\title{
A Review of Inflammatory Bowel Disease in Patients with Human Immunodeficiency Virus Infection
}

Avinash Adiga*, Deepa Panikkath and Kenneth Nugent

Texas Tech University Health Sciences Center, Lubbock, Texas, USA

\begin{abstract}
The coexistence of inflammatory bowel disease and human immunodeficiency virus infection is rare but does occur. Since immune mechanisms have an important role in the pathophysiology of both diseases, we might expect HIV infection to significantly alter the course of IBD. The pathophysiologic relationship between HIV and IBD is complex and controversial. Although many studies suggest spontaneous improvement in IBD symptoms and stable remissions after HIV infection, there are reports of relapse and new onset IBD with the decline in CD4 counts. Many of the opportunistic infections in HIV can mimic IBD and can lead to misdiagnosis and treatment as IBD. The treatment of IBD in HIV is associated with an increased risk of infections and lymphoma, and careful follow-up is essential.
\end{abstract}

Keywords: Human immunodeficiency virus; AIDS; Inflammatory bowel disease; Crohn's disease; Ulcerative colitis; Course; Diagnosis; Treatment; Immunosuppressive therapy; Surgery; HAART

\section{Introduction}

Human immunodeficiency virus (HIV) infection is the causative agent of acquired immunodeficiency syndrome, can result in a wide range of clinical consequences varying from asymptomatic disease to a life threatening opportunistic infections $[1,2]$. Constant viral replication results in depletion of peripheral and mucosal CD4 lymphocyte count and function causes secondary opportunistic infections leading to chronic diarrhea [3]. Gut associated lymphoid tissues (GALT) is an important location for HIV virus replication and pathogenesis, targeting mucosal CD4 lymphocytes [4]. It is suggested that the inflammation in inflammatory bowel disease (IBD) is initiated and propagated by an aggressive cell-mediated immune response to an unknown antigen in genetically susceptible host [5], GALT associated CD4 lymphocyte plays an important role in pathogenesis of IBD. HIV infection and IBD can coexist in the same person. As HIV is associated with decline in the immune system and IBD (which includes Crohn's disease and ulcerative colitis) is an inflammatory process, we might expect HIV to significantly alter the course of IBD in some patients. The relationship between IBD and HIV is complex and involves numerous interactions.

\section{Pathophysiology}

Inflammation in IBD is initiated and perpetuated by an aggressive cell-mediated immune response to unknown environmental antigens in a genetically susceptible host. Although Crohn's disease is a T- cell mediated disease, and ulcerative colitis (UC) is an antibody mediated disease, both conditions share many common end effector pathways. The etiology and specific mechanisms responsible for IBD are still poorly defined. The initiation of IBD and its pathogenesis are thought to be multifactorial, involving interactions among environmental, genetic, and immune factors.

GALT is the major lymphoid organ in the body with more than $50 \%$ of the total number of circulating $\mathrm{T}$ lymphocytes residing in the GALT. Among these, CD4+ T lymphocytes have an important role in the pathogenesis of human IBD; this association is supported by the clinical effects of monoclonal anti CD4+ antibodies in patients with Crohn's disease [6]. Inflammatory bowel disease patients with concomitant leukemia when treated with bone marrow transplantation can remain in long-term remissions, suggesting that the host immune dysregulation has an important role in the perpetuation of this disease [7]. Anti-TNF $a$ antibodies used in treatment of IBD can induce rapid mucosal T-cell apoptosis within 2 days, indicating that the therapeutic efficacy is due to the elimination of T-effector cells in the gut [8]. This evidence suggests that IBD is due to a dysregulation in the gut mucosal immune system, with activation of mucosal $\mathrm{T}$ lymphocytes and production of pro-inflammatory cytokines, such as tumor necrosis factor [5].

Studies reveal that with advanced HIV there are markedly reduced numbers of CD4 cells both in circulation and in the small bowel along with a parallel increase in CD8 cells [9]. A study on colonic biopsies in HIV patients revealed a significant increase in levels of inflammatory cytokines IL- $1 \beta$ and IFN $\Upsilon$ but a decrease in the levels of IL-10 [10]. When compared to patients with early stage disease, the peak expression of TNF $\alpha$ and IL- $1 \beta$ in rectal mucosa was higher in late stage disease [11]. In contrast to these findings, one study confirmed defective functioning of mononuclear cells in the colonic mucosa of AIDS patients, evidenced by reduction in TNF- $\alpha$ secretion [12]. In comparison to other organs, the number of HIV infected cells in the lamina propria has been consistently low [13].

The absolute CD4 cell count may not correlate with the course of IBD as it depends more on CD4 function than the absolute number [14]. Studies comparing patients with Crohn's disease with healthy controls showed no difference in the absolute number of CD4 cells in the lamina propria, but a higher proportion of T lymphocytes secreting IL-2 (indicating T-cell activation) was observed in patients with Crohn's disease. Decreases in peripheral CD4 counts may not imply intestinal mucosal immune depletion as the peripheral T- cell counts may not reflect the levels in the lamina propria [15]. In the presence of HIV,

*Corresponding author: Avinash Govinda Adiga, Affiliated to Department of Internal Medicine, Texas Tech University Health Sciences Center, Lubbock, Texas, USA, Tel: 8066202714; E-mail: dravinashadiga11@gmail.com

Received March 30, 2016; Accepted April 02, 2016; Published May 11, 2016

Citation: Adiga A, Panikkath D, Nugent K (2016) A Review of Inflammatory Bowe Disease in Patients with Human Immunodeficiency Virus Infection. J AIDS Clin Res 7: 575 . doi:10.4172/2155-6113.1000575

Copyright: () 2016 Adiga A, et al. This is an open-access article distributed under the terms of the Creative Commons Attribution License, which permits unrestricted use, distribution, and reproduction in any medium, provided the original author and source are credited. 
Citation: Adiga A, Panikkath D, Nugent K (2016) A Review of Inflammatory Bowel Disease in Patients with Human Immunodeficiency Virus Infection. J AIDS Clin Res 7: 575. doi:10.4172/2155-6113.1000575

Page 2 of 4

there is a decrease in all subsets of CD4 $\mathrm{T}$ cells, including those that mediate and prevent IBD, so the course of IBD will depend on the subset that is more affected. Inflammatory bowel disease might improve if the Th1 subset is disproportionately decreased, but IBD might worsen if the regulatory $\mathrm{CD} 4 \mathrm{~T}$ cell subset is disproportionately decreased. These data suggest a complex relationship between HIV and IBD. Since T cells have an important role in the pathogenesis of IBD, and HIV infection is characterized by destruction of CD4 T cells leading to immunodeficiency, the prevalence and natural history of IBD can be altered in patients with HIV infection. The possible pathophysiological relationship between IBD and HIV remains controversial. Some studies have suggested that the incidence of IBD in patients with HIV is increased, due to an increase in rectal inflammation or due to immune dysregulation induced by HIV, and that a decrease in the number and function of CD4 cells in HIV can facilitate the development of IBD [16]. Clinical studies have postulated that a progressive decline in CD4 counts caused by HIV may reduce disease activity in IBD and could contribute to remission [17-19].

\section{Course of Disease}

Studies have shown a significant decrease or no exacerbations of IBD when the CD4 count is decreased below 200 cells $/ \mathrm{mm}^{3}$ [20]. Several case reports reported spontaneous improvement in IBD symptoms and stable remissions after HIV infection and a decline in CD4 counts $[21,22]$. The time to first relapse is significantly longer in HIV patients when compared to normal individuals, and non- lymphocytopenic patients without immunosuppression relapsed more often than those on immunosuppression. A study that compared relapse rates between IBD patients with and without HIV concluded that the mean relapse rate for IBD patients with HIV was 0.016/year of follow-up while it was 0.053/year of follow-up for patients without HIV. Among the IBD and HIV patients, those who were on immunosuppressive treatment did not relapse, while $50 \%$ of patients who were not on immunosuppression had relapses on follow-up [23]. However, there are reports of new onset UC or Crohn's disease developing in patients with HIV infection/ AIDS with CD4 counts as low $100 / \mathrm{mm}^{3}[24,14]$ and of increases in IBD activity after the diagnosis of AIDS [25]. These reports concluded that HIV infection/AIDS does not have an effect on IBD. Another study which focused on new onset IBD in HIV positive patients revealed that absolute CD4 count of patients with "de novo" IBD was 210-700cells/ $\mathrm{mm}^{3}$ at the time of diagnosis [24]. Similar reports describing "de novo" IBD in HIV positive patients commented that IBD activity is independent of HIV/AIDS; the CD4 counts in these patients were 480500 cells $/ \mathrm{mm}^{3}$ or greater [25]. Researchers at Digestive Disease Week meeting in San Diego in 2008 concluded that the incidence of ulcerative colitis in HIV is about double that expected in a normal population. They also added that there is no clear association with CD4 counts and the time of diagnosis of IBD. Interestingly, patients with chronic colon inflammation had accelerated CD4 cell depletion and a slight increase in CD4 counts was noted with colectomy [26] (Figure 1).

\section{Diagnostic Difficulties}

The diagnosis of IBD in HIV patients is quite challenging since gastrointestinal disorders occur frequently in patients with AIDS. More than $50 \%$ of the patients have diarrhea at some point during the course of HIV and AIDS. Although both UC and Crohn's disease has been described in HIV, opportunistic infections and neoplasms comprise the majority of colonic disorders in these patients. A distinct form of IBD called "idiopathic inflammatory disease of the colon" has also been described [27].

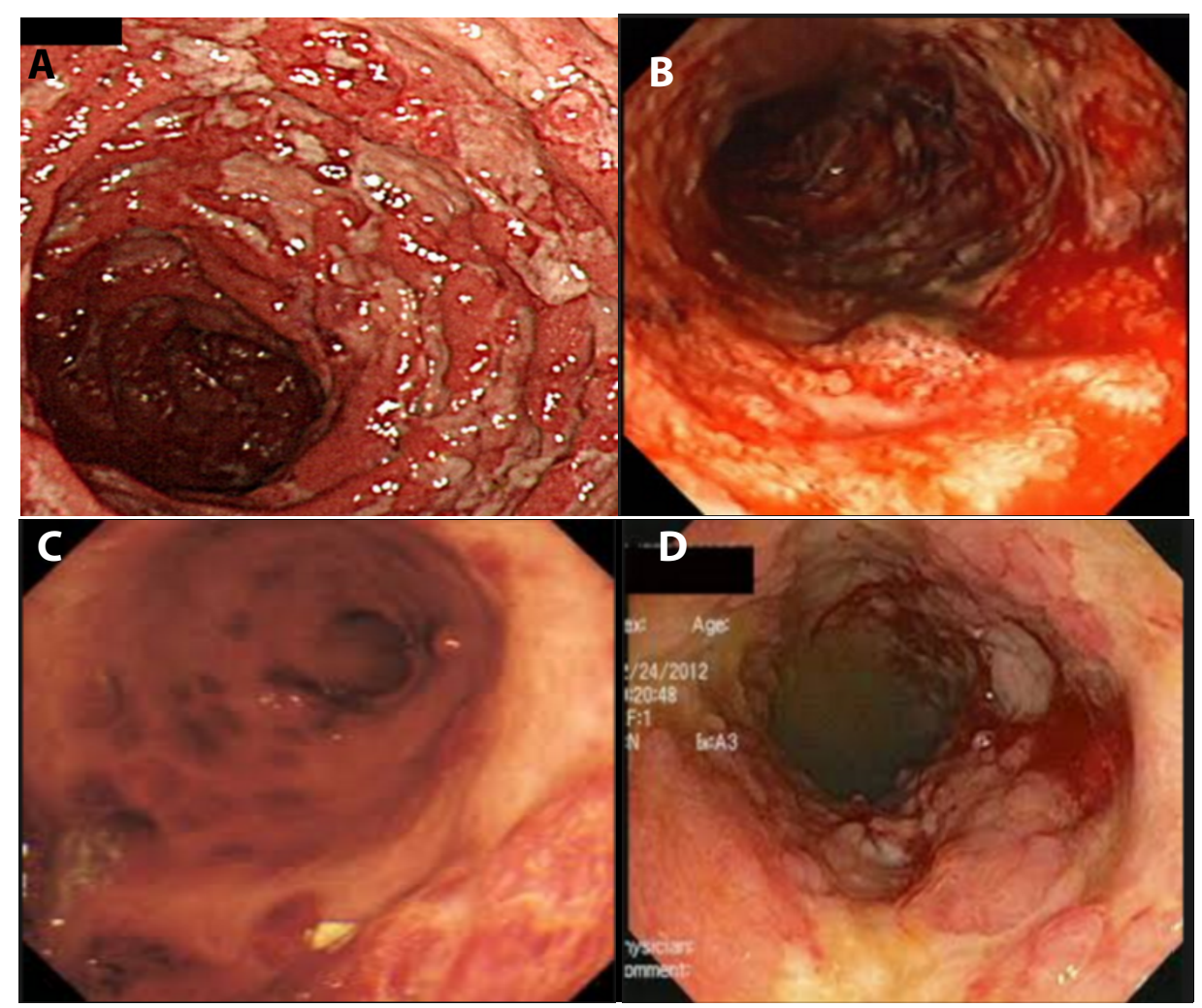

Figure 1: Endoscopic findings in A: CMV colitis, B: Ulcerative colitis, C: Kaposi sarcoma, D: Infectious colitis (clockwise from top left). 
Citation: Adiga A, Panikkath D, Nugent K (2016) A Review of Inflammatory Bowel Disease in Patients with Human Immunodeficiency Virus Infection. J AIDS Clin Res 7: 575. doi:10.4172/2155-6113.1000575

Page 3 of 4

Many opportunistic infections can mimic IBD clinically, endoscopically, and pathologically and can be misdiagnosed as chronic IBD [28]. The term "AIDS enteropathy" has been described when no organisms can be isolated from stool samples and is usually caused by pathogens like cryptosporidium [29]. Enteritis and colitis due to cytomegalovirus can endoscopically mimic IBD, but biopsies will show profound mucosal ulcerations and transmural fibrosis without granulomas [30]. Gastrointestinal Kaposi sarcoma presents with extensive submucosal infiltration, and the overlying mucosal inflammation can resemble UC [31]. Soria et al. reported a case series in which IBD was misdiagnosed and treated in patients with HIV when the histologic definition was not conclusive and lacked the typical IBD pattern [32]. It is claimed that all cases of IBD in HIV infection need biopsy confirmation. European Crohn's and colitis organization guidelines recommend that HIV testing should be done in all patients before prescribing prednisone as a treatment for IBD.

\section{Therapeutic Options}

A confirmatory biopsy suggestive of IBD is necessary before starting treatment for de-novo IBD in the setting of HIV. Tolerance of anti-inflammatory therapy and secondary infections are limiting factors to therapy due to immunocompromised state. Drug allergies to sulfonamide are more common in patients with HIV infection, but reactions to sulfasalazine do not seem to be more common. Sulfasalazine is generally well tolerated, and there are no major drug interactions with HIV medications. Methotrexate was associated with major infections in several case reports in the pre-ART era [33] but seems to be well tolerated in well controlled HIV [34]. Immunosuppressive drugs like mycophenolate, azathioprine, and cyclosporine have been used without major short-term toxicity [35]. Longer-term use increases the risk of infection and lymphoma, and the risk of lymphoma remains increased even in the ART era. Corticosteroids are relatively safe and well tolerated in short-term use in HIV infection. Long term use is associated with increased risk of infection and the metabolic consequences of corticosteroid use (like bone loss) that have the potential to exacerbate the metabolic syndrome characteristic of chronic HIV [36]. TNF inhibitors (infliximab and etanercept) are usually tolerated well and safely used [16], but the strong association between HIV and tuberculosis suggests an increased risk of mycobacterial and other intracellular infections. Apart from immunodeficiency due to HIV, treatment of IBD with immunosuppressive drugs significantly increases the risk of secondary infection. Prevention of infection is crucial in these patients, which includes recognition of risk factors, monitoring for clinical symptoms, attention to laboratory results, vaccination and patient education. A decrease in secondary infection rate is observed with lower dose, shorter course and with night-time dosing of corticosteroids and in patients with less comorbidity [35]. Regular monitoring of laboratory results in patients on methotrexate and thiopurines for leukopenia is necessary. Advanced age and concurrent steroids at the start of antiTNF therapy are predictors of secondary infection [36].

Surgery is reserved for those patients who cannot tolerate medical management or when complications like bleeding, perforation, or severe infection arise. HIV-infected patients without AIDS-defining criteria have a surgical course similar to that of noninfected patients but the post procedural complication rates are higher in anorectal surgeries. HIV and AIDS are considered as independent risk factors for surgery and end-stage disease must be considered as a comorbidity. Surgery should be tailored accordingly [37]. Studies have revealed that HAART therapy had no influence on IBD relapse [23].

\section{Conclusion}

Despite several studies and reports, the exact course of IBD in HIV infection remains inconclusive. Due to the absence of experimental models, additional clinical studies are needed to better understand the pathophysiologic mechanisms. A diagnosis of IBD in HIV needs extreme caution as many opportunistic infections seen in HIV can mimic IBD, and this diagnosis requires confirmatory findings on biopsy. Therapeutic options should be tailored individually due to increased risk of secondary infections and lymphomas.

\section{References}

1. Fanci A (2006) Twenty five years of HIV-AIDS. Science 313: 409.

2. Sierra S, Kupfer B, Kaiser R (2005) Basics of the virology of HIV-1 and its replication. J Clin Virol 34: 233-244.

3. McMichael AJ, Rowland-Jones SL (2001) Cellular immune responses to HIV Nature 410: 980-987.

4. Brenchley JM, Douek DC (2008) HIV infection and the gastrointestinal immune system. Mucosal Immunol 1: 23-30.

5. Shih DQ, Targan SR, McGovern D (2008) Recent advances in IBD pathogenesis: genetics and immunobiology. Curr Gastroenterol Rep 10: 568-575.

6. Emmrich J, Seyfarth M, Fleig WE, Emmrich F (1991) Treatment of inflammatory bowel disease with anti-CD4 monoclonal antibody. Lancet 338: 570-571.

7. Lopez-Cubero SO, Sullivan KM, McDonald GB (1998) Course of Crohn's disease after allogeneic marrow transplantation. Gastroenterology 114: 433440 .

8. ten Hove T, van Montfrans C, Peppelenbosch MP, van Deventer SJ (2002) Infliximab treatment induces apoptosis of lamina propria $\mathrm{T}$ lymphocytes in Crohn's disease. Gut 50: 206-211.

9. Rodgers VD, Fussett R, Kagnoff MF (1986) Abnormalities in intestinal mucosa $T$ cells in homosexual populations including those with the lymphadenopathy syndrome and acquired immunodeficiency syndrome. Gastroenterology 90 : $552-558$

10. McGowan I, Radford-Smith G, Jewell DP (1994) Cytokine gene expression in HIV-infected intestinal mucosa. AIDS 8: 1569-1575.

11. Reka S, Garro ML, Kotler DP (1994) Variation in the expression of human immunodeficiency virus RNA and cytokine mRNA in rectal mucosa during the progression of infection. Lymphokine Cytokine Res 13: 391-398.

12. Steffen M, Reinecker HC, Peterson J (1993) Differences in cytokine secretion by intestinal mononuclear cells, peripheral blood macrophages from human immunodeficiency infected patients. Clinical Experimental Immunology 91: 30 36.

13. Jarry A, CortezA, Rene E, Muzeau F, Brouse N (1990) Infected cells and immune cells in the gastrointestinal tract of AIDS patients. An immunohistochemical study of 127 cases. Histopathology 16: 133-140.

14. Lautenbach E, Lichtenstein GR (1997) Human immunodeficiency virus infection and Crohn's disease: the role of the CD4 cell in inflammatory bowel disease. Journal Clinical Gastroenterology 25: 456-459.

15. Bernstein CN, Snape WJ Jr (1991) Active idiopathic ulcerative colitis in a patient with ongoing HIV-related immunodepression. Am J Gastroenterol 86: 907-909.

16. Himmel ME, Hardenberg G, Piccirillo CA, Steiner TS, Levings MK (2008) The role of T-regulatory cells and Toll-like receptors in the pathogenesis of human inflammatory bowel disease. Immunology 125: 145-153.

17. Pospai D, René E, Fiasse R (1998) Crohn's disease stable remission after human immunodeficiency virus infection. Digestive Disease Sciences 43: 412419.

18. James SP (1988) Remission of Crohn's disease after human immunodeficiency virus infection. Gastroenterology 95: 1667-1669.

19. Dhar JM, Pidgeon ND, Burton AL (1984) AIDS in a patient with Crohn's disease. Br Med J (Clin Res Ed) 288: 1802-1803.

20. Christ AD, Sieber CC, Cathomas G (1996) Concomitant active Crohn's disease and the acquired immunodeficiency syndrome. Scandinavian Journal Gastroenterology 31: 733-735. 
Citation: Adiga A, Panikkath D, Nugent K (2016) A Review of Inflammatory Bowel Disease in Patients with Human Immunodeficiency Virus Infection. J AIDS Clin Res 7: 575. doi:10.4172/2155-6113.1000575

Page 4 of 4

21. Bernstein BB, Gelb A, Tabanda-Lichauco R (1994) Crohn's ileitis in a patient with longstanding HIV infection. Am J Gastroenterol 89: 937-939.

22. Sturgess I, Greenfield SM, Teare J, O'Doherty MJ (1992) Ulcerative colitis developing after amoebic dysentery in a haemophiliac patient with AIDS. Gut 33: 408-410.

23. Viazis N, Vlachogiannakos J, Georgiou O (2010) Course of inflammatory bowel disease in patients infected with human immunodeficiency virus. Inflammatory Bowel Disease 16: 507-511.

24. Yoshida E, Chan N, Herrick R, Amar JN, Sestak PM, et al. (1996) Human immunodeficiency virus infection, the acquired immunodeficiency syndrome and inflammatory bowel disease. Journal of Clinical Gastroenterology 23: 24-28.

25. Liebowitz D, McShane D (1986) Nonspecific chronic inflammatory bowel disease and AIDS. J Clin Gastroenterol 8: 66-68.

26. J Landy, B Gazzard, M Harbord. Inflammatory Bowel Disease in HIV Seropositive Individuals: Analysis of a Large Cohort. Gastroenterology 134: A-504

27. Charles Mel Wilcox (2015) Human immunodeficiency virus and inflammatory bowel disease. Inflammatory Bowel Disease: From Bench to Bedside 863-873.

28. Fanci A (2006) Twenty five years of HIV-AIDS. Science 31:409.

29. Smith PD (1993) Infectious diarrheas in patients with AIDS. Gastroenterol Clin North Am 22: 535-548.
30. Knapp AB, Horst DA, Eliopoulos G, Gramm HF, Gaber LW (1983) Widespread cytomegalovirus gastroenterocolitis in a patient with the acquired immunodeficiency syndrome. Gastroenterology 85: 1399-1402.

31. Weber JN, Carmichael DJ, Boylston A, Munro A, Whitear WP, et al. (1985) Kaposi's sarcoma of the bowel--presenting as apparent ulcerative colitis. Gut 26: $295-300$.

32. Soria A, Rossi M, Muscatello A, Gori A (2011) HIV testing: a must for patients with inflammatory bowel disease? Am J Gastroenterol 106: 1727-1728.

33. Winchester RJ (1994) HIV infection and rheumatic disease. Bull Rheum Dis 43: 5-8.

34. Norbiato G (2012) Endocrine, metabolic, and immunologic components of HIV infection. Ann N Y Acad Sci 1262: 51-55.

35. Stuck AE, Minder CE, Frey FJ (1989) Risk of infectious complications in patients taking glucocorticosteroids. Rev Infect Dis 11: 954-963.

36. Toruner M, Loftus EV Jr, Harmsen WS, Zinsmeister AR, Orenstein R, et al. (2008) Risk factors for opportunistic infections in patients with inflammatory bowel disease. Gastroenterology 134: 929-936.

37. Foo E, Sim R, Lim HY, Chan ST, Leo YS, Wong SY (1998) Abdominal surgery in HIV infected patients- early local experience. Ann Acad Med Singapore 27 759-762. 